\title{
New horizons for multiple sclerosis therapeutics: milestones in the development of ocrelizumab
}

This article was published in the following Dove Press journal:

Neuropsychiatric Disease and Treatment

\section{Jessica Frau* \\ Giancarlo Coghe* \\ Lorena Lorefice \\ Giuseppe Fenu \\ Eleonora Cocco}

Multiple Sclerosis Center Binaghi Hospital, Department of Medical

Sciences and Public Health, University of Cagliari, Cagliari, Italy

*These authors contributed equally to this work
Correspondence: Jessica Frau Multiple Sclerosis Center Binaghi Hospital, Department of Medical Sciences and Public Health, University of Cagliari,

Via Is Guadazzonis, 2, 09126 Cagliari, Italy Tel +390706092929

Email jessicafrau@hotmail.it

\begin{abstract}
Multiple Sclerosis (MS) is an inflammatory and neurodegenerative disease of the central nervous system, and both $\mathrm{T}$ and $\mathrm{B}$ cells are involved in its pathogenesis. The vast majority of disease-modifying drugs used for MS act on the inflammatory component of the disease and are approved for use in relapsing-remitting (RR) patients. Ocrelizumab (OCR) is the only MS drug that has been approved by the US Food and Drug Administration (FDA) not only for patients with RRMS but also for patients with primary progressive (PP) MS. OCR is a humanized anti-CD20 monoclonal antibody that can deplete the targeted B cells through antibody-dependent cellular cytotoxicity. Treatment involves administration by intravenous infusion every 6 months. OCR can cause long-lasting B-cell depletion and change the pool of reconstituted B cells. Phase III clinical trials have confirmed the results of previous Phase II studies. In particular, OPERA I and II trials, which were performed in patients with RRMS, showed a reduction in the annualized relapse rate, the risk of disability progression, and the number of new/enlarging T2 lesions and enhancing lesions measured using brain magnetic resonance. The ORATORIO trial, performed in PP subjects, showed that OCR can reduce disability progression, improve performance on the timed 25-foot walk, and decrease the total volume of $\mathrm{T} 2$ lesions and the mean number of new or enlarging $\mathrm{T} 2$ lesions. The most frequent adverse events were the infusion-related reactions and infections. Infections were mostly nasopharyngitis, as well as upper respiratory and urinary tract infections. OCR gives no indication for severe or opportunistic infections. There is not a clear increased risk of malignancies. Nevertheless, it could not be excluded. Real-life registries will provide more information about the long-term safety, the risk of exposure during pregnancy, and the risk of rare adverse events. In this review, we analyze the evidence regarding the efficacy and the safety of OCR.
\end{abstract}

Keywords: multiple sclerosis, ocrelizumab, anti-CD20 antibody

\section{Introduction}

Multiple sclerosis (MS) is an inflammatory and neurodegenerative disease of the central nervous system. At the onset of the disease, the most common clinical course is the relapsing-remitting $(\mathrm{RR})$ phenotype, while a minority of patients experience an immediate progression of disability, referred to as the primary progressive (PP) phenotype. After initial relapsing course, the patients would eventually shift to a secondary progressive course in which there is a progressive accumulation of disability over time. ${ }^{1}$

The clinical manifestations of MS indicate the involvement of various functional systems: motor, sensory, visual, bowel-bladder, cognitive, brainstem, and cerebellar. Moreover, frequent features of the disease are the fatigue and the Uhthoff phenomenon, that is, a transient worsening of symptoms secondary to an increase of body temperature (fever, physical exercise, hot bath, and so on).

In clinical practice as well as in clinical trials, the level of disability is assessed using the expanded disability status scale (EDSS), which ranges from 0 (absence of deficits) 
to 10 (death due to MS). This scale addresses all of the functional systems that could be involved in the disease, but is mainly focused on ambulation autonomy. ${ }^{2}$

Both $\mathrm{T}$ and $\mathrm{B}$ cells are involved in the pathogenesis of $\mathrm{MS}$, and the results of their action are demyelination, axonal loss, and gliosis. ${ }^{3}$ The role of B cells is supported by various factors, such as their presence in MS plaques ${ }^{4,5}$ and in the meningeal follicles of secondary progressive patients, ${ }^{6}$ and the frequent finding of oligoclonal bands (OCBs) in the cerebrospinal fluid (CSF) of patients with $\mathrm{MS}{ }^{7}$ OCBs are secreted by B cells and plasma cells and their presence is nearly stable in the same subject over the course of the disease; thus, OCBs are considered to be a hallmark of each person with MS. ${ }^{8,9}$ Another piece of evidence supporting the role of B cells in MS derives from the identification of some B-cell-specific genes shown to increase the risk of MS, perhaps due to their capacity to shift B cells into a proinflammatory profile. ${ }^{10}$ Recently, an association between a variant of the TNFSF $13 B$ gene, encoding for the cytokine "B-cell activating factor" (BAFF), and an increased risk of MS has been found. ${ }^{11}$ BAFF is essential for B-cell activation, differentiation, and survival. ${ }^{12}$ Further, its levels were found to be modified by treatments used in MS, such as interferon beta and methylprednisolone. ${ }^{13,14}$ These pieces of evidence support the importance of therapies that target B cells.

To date, many disease-modifying drugs have been studied and employed in the treatment of MS, ${ }^{15}$ with the aim of reducing aspects of the disease related to inflammation, clinical relapses, new T2 lesions, and gadolinium-enhancing lesions on brain and spinal cord magnetic resonance imaging (MRI). Moreover, there are other emerging immunotherapeutic strategies for MS. Among them, the use of stem cells is the most studied. In particular, the hematopoietic stem cell transplantation has been used from many years in the experimental treatment of MS, while the use of mesenchymal stem cells is more recent. There are also some Phase I and II studies investigating the potential role of the BHT-3009 DNA vaccine and the altered peptide ligands. ${ }^{15}$

All of the medications are actually disposable and are only suitable for patients with the RR clinical course. However, recently, ocrelizumab (OCR) has been approved by the US FDA to treat both RR and PP patients (March 2017).

\section{Use of OCR in MS: rational and mechanism of action}

OCR is a monoclonal antibody with an IgG1 tail that is able to bind to a specific epitope of CD20, which is different than those bound by rituximab and ofatumumab. ${ }^{16}$
These three antibodies differ in their structure; OCR is humanized, rituximab is mouse-human, and ofatumumab is fully human ${ }^{17}$ (Figure 1). Their target molecule (CD20) is a glycosylated phosphoprotein that is expressed in the vast majority of B-cell lines, but not in stem cells, pro-B cells, and plasma cells ${ }^{18}$ (Figure 2 ). The plasma cells being the most important producers of antibodies in the vast majority of subjects, the levels of antibodies in blood and CSF are generally not reduced after anti-CD20 therapies, while B cells are depleted via three principal mechanisms: antibody-dependent cellular cytotoxicity, complementdependent cytotoxicity, and apoptosis. ${ }^{19-22}$ Moreover, OCR acts also in a subtype of circulating $\mathrm{T}$ cells that express CD20, representing $\sim 6 \%$ of total T cells. ${ }^{23}$ OCR's action is mostly mediated by antibody-dependent cellular cytotoxicity, while the anti-CD20 antibodies rituximab and ofatumumab by complement-dependent cytotoxicity ${ }^{10}$ (Figure 3 ). B-cell depletion is very important for the suppression of inflammation in MS. Indeed, these cells are "bad" because they can secrete cytokines, selectively present antigens to T cells, and produce antibodies together with plasma cells. ${ }^{24}$ Moreover, the meningeal lymphoid follicles could be associated with cortical demyelination and axonal $10 \operatorname{loss}^{25,26}$ On the other hand, B cells are also "good", in that they are involved in cellular growth, remodeling, and repair. Moreover, regulatory B cells are important in the control of excessive inflammation. ${ }^{10}$

A depletion of $\sim 99 \%$ of B cells occurred within the first 14 days after infusion of OCR and persisted until week 24. ${ }^{27}$ B-cell depletion could be monitored by the assessment of CD19 cells. ${ }^{28}$ Afterward, B-cell reconstitution starts gradually, with a median recovery time of 74 weeks. In a minority of cases, the depletion continues 2.5 years after the last infusion. ${ }^{29}$ Anti-CD20 therapies are able to permanently change the pool of reconstituted B cells to produce predominantly naïve B cells and diminished plasmablasts and memory B cells. It has been demonstrated that anti-CD20 drugs also change the $\mathrm{T}$-cell repertoire after treatment, becoming more

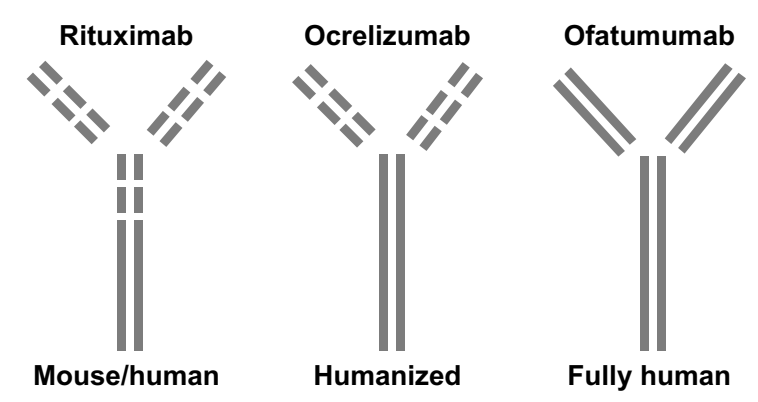

Figure I Representation of the three anti-CD20 monoclonal antibodies: rituximab, ocrelizumab, and ofatumumab. 


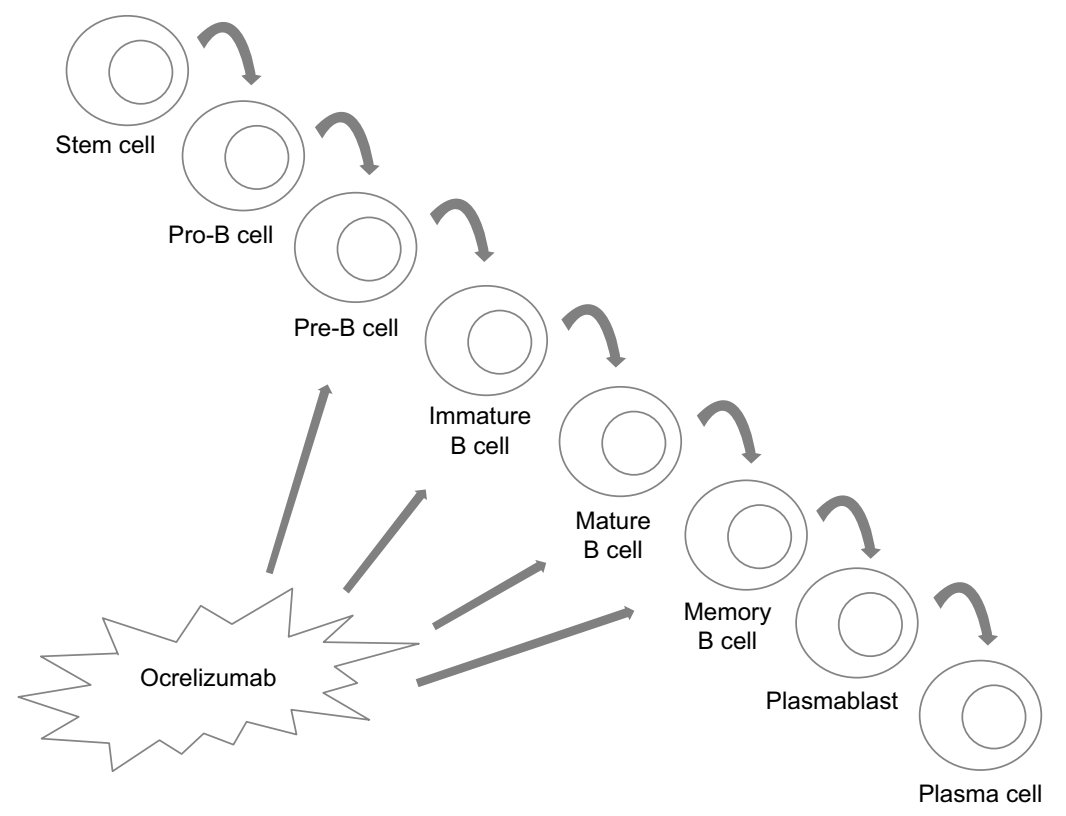

Figure 2 B-cell lines that are targeted and spared by the action of ocrelizumab.

represented by regulatory $\mathrm{T}$ cells than by pro-inflammatory $\mathrm{T}$ helper 1 and T helper 17 cells. ${ }^{30}$ However, a decrease of $\mathrm{T}$ cells in the first 6 months after treatment with anti-CD20 drugs has been observed. ${ }^{31}$ This global reprogramming of the immune system could explain the long-lasting effects of anti-CD20 treatments, even after immune reconstitution. ${ }^{32}$

In addition to anti-CD20 antibodies, other treatments with a prevalent action on $\mathrm{B}$ cells are under evaluation. The antibody inebilizumab is directed against the antigen CD19, which is expressed on a broader spectrum of B-lineage cells, comprising pro-B cells and plasmablasts. A Phase I randomized clinical study is ongoing to investigate the safety

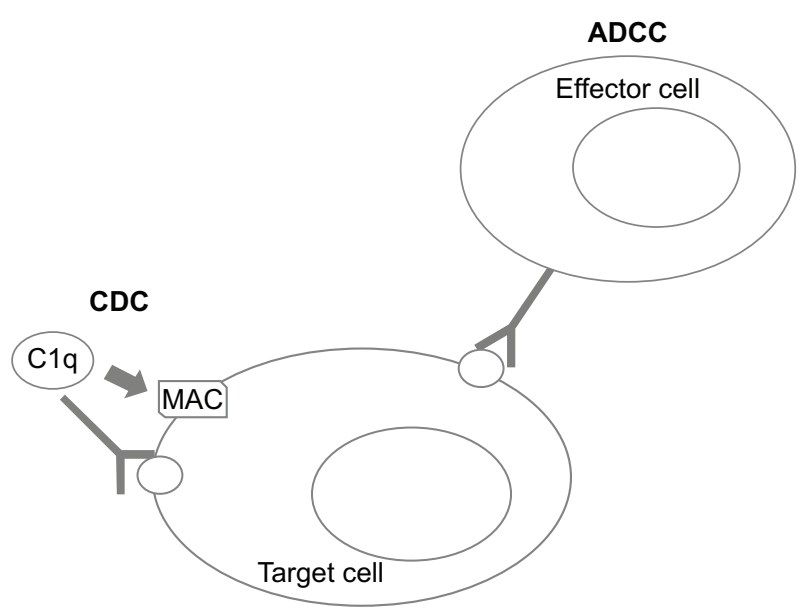

Figure 3 Mechanism of antibody-dependent cellular cytotoxicity (ADCC) and complement-dependent cytotoxicity (CDC). Abbreviaiton: MAC, membrane attack complex. of inebilizumab in patients with RRMS. Other important targets are the blockers of BAFF (belimumab, LY2127399, and VAY736) and of "a proliferation-inducing ligand" (atacicept). In fact, they are members of TNF superfamily and are important for maturation and function of B cells. Some Phase II clinical trials investigating their role on clinical and MRI features in subjects with RRMS are ongoing. ${ }^{33}$

\section{Efficacy data from clinical trials}

Clinical data about the efficacy of OCR in patients with RRMS are derived from three clinical trials. ${ }^{27,28,34}$ The first one was a Phase II, randomized, placebo-controlled study, in which 220 patients were enrolled from 79 centers in 20 countries and divided into four groups. ${ }^{27}$ All of the subjects had an aggressive course of disease, with at least three relapses in the 3 years before the baseline and at least one in the past year. Two groups received OCR treatment, which was administered by four intravenous infusions over 24 weeks, at a dose of 600 or $2,000 \mathrm{mg}$. Regarding the other two groups, one received placebo infusions and the other received interferon beta 1a intramuscular injections once a week. Before each infusion, intravenous methylprednisolone $100 \mathrm{mg}$ was administered, and analgesic or antipyretic with oral antihistamine were recommended to avoid infusionrelated adverse events. Out of the whole cohort, 204 subjects completed the 24-week period of the study and $196 \mathrm{com}$ pleted the 48-week period. Both groups treated with OCR had an overall relative reduction of gadolinium-enhancing 
lesions compared to the placebo group (89\% for the $600 \mathrm{mg}$ group and $96 \%$ for the $2,000 \mathrm{mg}$ group). The patients free of gadolinium-enhancing lesions were $77 \%$ in the $600 \mathrm{mg}$ group and $88 \%$ in the $2,000 \mathrm{mg}$ group. Moreover, the annualized relapse rate (ARR) over 24 weeks was lower in the OCR treated groups than in the placebo group (80\% lower in the $600 \mathrm{mg}$ group and $73 \%$ lower in the $2,000 \mathrm{mg}$ group). The effect of reducing clinical relapses persisted between 24 and 48 weeks.

After the positive results of the Phase II study, two Phase III, multicenter, randomized, double-blind, active-controlled, parallel group trials were performed in RR patients: OPERA I and OPERA II. ${ }^{28}$ They were conducted at independent sites and had the same protocol design, with OCR $600 \mathrm{mg}$ administered to the study group and interferon beta $1 \mathrm{a} 44 \mu \mathrm{g}$ subcutaneous injections three times/week to the control group. The infusion protocol was the following: an infusion every 24 weeks, with the first $600 \mathrm{mg}$ dose divided into two infusions, each of $300 \mathrm{mg}$, administered on days 1 and 15; the following infusions were single $600 \mathrm{mg}$ administrations. The OPERA I trial involved 821 patients from 141 sites in 32 countries, while the OPERA II trial involved 835 patients from 166 sites in 24 countries. The principal inclusion criteria were as follows: age 18-55 years; EDSS score 0-5.5; and at least two relapses in the 2 years before the study, or 1 relapse in the past year. Similar to that in the Phase II trial, intravenous $100 \mathrm{mg}$ of methylprednisolone was administered before each infusion, and analgesic or antipyretic with oral antihistamine were recommended. The primary end point was the ARR at 96 weeks, and the study had many secondary end points: the proportion of patients with disability progression confirmed at 12 weeks; disability improvement confirmed at weeks 12 through 96; gadolinium-enhancing lesions, new or newly enlarged T2 lesions, new hypointense T1 lesions, and changes in brain volume measured with MRI; change in MS functional composite score; change in short-form health survey (SF-36). OPERA I was completed by $89.3 \%$ patients in the OCR group and $82.7 \%$ in the interferon beta group, while OPERA II was completed by $86.3 \%$ and $76.6 \%$, respectively. In both studies, the patients treated with OCR had a lower ARR at 96 weeks than those treated with interferon beta ( 0.16 versus 0.29 ). Thus, the ARR was $46 \%$ lower in the OCR group $(p<0.001)$. Furthermore, patients treated with OCR had a $40 \%$ lower risk of disability progression at 12 and 24 weeks after treatment $(p<0.001$ and $p=0.003$, respectively). In OPERA I, a significant effect on the rate of disability improvement was also shown at 12 weeks, being 33\% higher in the OCR than in the interferon beta group $(p=0.02)$.
MRI analysis showed a 94\% (OPERA I) and 95\% (OPERA II) reduction in the number of gadolinium-enhancing lesions with OCR than with interferon beta $(0.02$ versus $0.29, p<0.001$ in OPERA I; 0.02 versus $0.42, p<0.001$ in OPERA II). Moreover, patients treated with OCR had a lower number of new or newly enlarging T2 lesions than patients treated with interferon beta in both OPERA I (77\%, $p<0.001 ; 0.32$ versus 1.41 , respectively) and OPERA II ( $83 \%, p<0.001 ; 0.33$ versus 1.90 , respectively). Most of the new or newly enlarged T2 lesions in patients treated with OCR developed within the first 24 weeks. Compared to the interferon beta group, lesions were 94\% (OPERA I) and $96 \%$ (OPERA II) lower from week 24 to week 48, while 98\% (OPERA I) and 97\% (OPERA II) lower from week 48 to week 96 in the OCR group.

The results about changes in the SF-36 score were not conclusive. Indeed, in both studies on RR patients, SF-36 scores were better with OCR than with interferon beta, but the result was statistically significant only in OPERA II.

As mentioned, OCR is the first drug approved for patients with the PP course of MS. The interest in studying this therapy in these subjects arises from the results of the OLYMPUS clinical trial, which investigated the efficacy of the anti-CD20 antibody rituximab in patients with PPMS. ${ }^{35}$ The results of this study were considered negative, because the primary end point was not reached. Nevertheless, after the analysis of subgroups, younger subjects ( $<51$ years old) with evidence of disease activity demonstrated a delay in disability progression.

The ORATORIO study ${ }^{34}$ was a Phase III, randomized, parallel-group, double-blind, placebo-controlled trial, investigating the efficacy of OCR in 732 patients with PPMS and specific clinical features. The inclusion criteria were very strict, and included the following: age 18-55 years; an EDSS score between 3.0 and 6.5; a score in the pyramidal functional system of the EDSS of at least 2; a short duration of disease (less than 15 years if the EDSS score was more than 5.0, and less than 10 years if the EDSS score was 5.0 or less); an elevated IgG index or at least one OCB in CSF. The modality of drug administration and the prophylaxis recommended against infusion-related reactions were identical to those in the previous trials performed in RR subjects. The patients assigned to OCR and who completed the study (120 weeks) were 402 out of 488 .

The primary end point of this study was the percentage of patients with disability progression confirmed at 12 weeks. The study also had some secondary end points: the percentage of patients with disability progression confirmed at 24 weeks; the change in total volume of $\mathrm{T} 2$ brain lesions and in brain 
volume; the change in performance on the timed 25 -foot walk; and the change in the SF-36 score.

The primary end point was reached, showing a $24 \%$ reduction of disability progression in the active group $(p=0.03)$. The disability progression at 12 weeks was $32.9 \%$ with OCR and $39.3 \%$ with placebo. Similar results were found for the first secondary end point: the disability progression at 24 weeks was $29.6 \%$ with OCR and $35.7 \%$ with placebo, with a lower risk of $25 \%$ for the active group ( $p=0.04)$.

The drug was also more effective in improving the performance on the timed 25-foot walk compared to placebo (change of $38.9 \%$ versus $55.1 \%$, respectively, relative reduction with OCR of $29.3 \%, p=0.004$ ).

The MRI end point results were once again positive. Indeed, a decrease in the total volume of $\mathrm{T} 2$ lesions from baseline to week 120 with OCR was observed, while there was an increase with placebo (mean percent change -3.4 , $p<0.001$ ). The mean change in brain volume from week 24 to week 120 was -90 with OCR and -1.09 with placebo ( $p=0.02$ ). Moreover, the mean number of new or enlarging $\mathrm{T} 2$ lesions from baseline to week 120 was 0.31 with OCR and 3.88 with placebo $(p<0.001)$.

\section{Safety data from clinical data Infusion reactions}

The principal adverse events observed were infusion reactions and infections. The infusion reactions were mild or moderate, occurred mostly during the first dose, and were mainly pruritus, rash, throat irritation, and flushing. One case of life-threatening bronchospasm occurred in the OPERA I trial during the first infusion. All the remaining infusion reactions were not life-threatening and none were fatal. ${ }^{27,28,34}$

\section{Infections}

The other frequent adverse event was the occurrence of infections, which were observed in similar percentages in both the OCR and interferon groups (56.9\% versus $54.3 \%$ in OPERA I; $60.2 \%$ versus $52.5 \%$ in OPERA II, respectively). ${ }^{28}$ Furthermore, in the ORATORIO trial, the percentage of infections was similar between the OCR and placebo groups (71.4\% versus $69.9 \%$, respectively), as well as the percentage of serious infections ( $6.2 \%$ versus $5.9 \%$, respectively). The OCR-exposed patients enrolled in ORATORIO trial had more frequent infections of oral herpes compared to the placebo group. ${ }^{34}$

The most frequent infections observed during the clinical trials were nasopharyngitis, as well as upper respiratory and urinary tract infections.
The selectivity of OCR could explain the similar rate of infection to that of the control groups. Indeed, humoral immunity and the immune reconstitution are maintained after the treatment, because B-cell precursors and plasma cells are spared from OCR's effects.

Moreover, a higher risk of either opportunistic or general serious infections in MS patients has not been reported. ${ }^{27,28,34}$ A clinical trial to treat rheumatoid arthritis using a high dose of OCR as an add-on treatment was stopped due to opportunistic infections. ${ }^{36}$

There were no cases of progressive multifocal leukoencephalopathy (PML) that were reported during OCR in the clinical trials. In May 2017, the first case of PML observed during OCR has been described. This patient received the first dose of OCR in April 2017, and the last dose of natalizumab in February 2017, after being on this drug for 3 years. This case was interpreted as "carryover" effect of natalizumab, which is notoriously associated with PML. In fact, the patient was exposed for a long period to natalizumab, and this drug was stopped only a few months before the diagnosis of PML, and only one dose of OCR was administered. ${ }^{37}$

Patients with both active $B$ and $C$ hepatitis could not be treated with OCR, as well as with other anti-CD20 antibodies, until infection is resolved. ${ }^{10}$ In subjects positive for hepatitis B core antibody and negative for the other hepatitis B virus markers (comprising absence of viral DNA), an hepatology evaluation and a prophylaxis with antiviral drugs should be considered. ${ }^{17}$

\section{Pregnancy}

Contraception is recommended for women in the 6 months after the last infusion. Indeed, in primates, the exposure to B-cell-depleting drugs was related to B-cell depletion in new borns, renal and testicular toxicity, lymphoid follicle formation in the bone marrow, and death. ${ }^{17}$ In children of women treated with B-cell depleting therapies during pregnancy, B-cell depletion and lymphocytopenia have been reported. ${ }^{17}$ There are no specific data about exposure to OCR.

\section{Kidney and hepatic failure}

The safety of OCR in patients with mild, but not severe, kidney and hepatic failure has been assessed in clinical trials. Thus, the drug is not contraindicated in subjects with mild kidney and hepatic dysfunction..$^{27,28,34}$

\section{Malignancies}

During the OPERA studies and their extension phase, nine neoplasms were detected in OCR group: four instances of 
breast cancer, one of renal cell carcinoma, two of malignant melanoma, and two of basal-cell skin carcinoma. There were two malignancies in the placebo group. ${ }^{27,28}$ Among the 486 patients enrolled in the ORATORIO study, a malignancy was observed in 11 patients exposed to OCR (2.3\%): four breast cancers, three basal-cell carcinomas, one endometrial adenocarcinoma, one anaplastic large-cell lymphoma, one malignant fibrous histiocytoma, and one pancreatic carcinoma. $^{34}$

Considering the clinical trials together, the overall incidence of a first neoplasm in patients treated with OCR was 0.40 per 100 patient-years of exposure, while in the whole cohort, including placebo and interferon beta, the incidence was 0.20 per 100 patient-years of exposure..$^{27,28,34}$

The number of OCR-exposed patients who had cancer diagnosis was not higher than expected in the general population, and the malignancies occurred were various. Also the breast cancers, which were the more represented, were of different types. Due to these considerations, a clear causative role of OCR exposure has not been clearly confirmed. ${ }^{10}$

\section{Severe adverse events}

The percentage of serious adverse events was lower in the OCR group than in the interferon group (6.9\% versus $7.8 \%$ in OPERA I, and $7.0 \%$ versus $9.6 \%$ in OPERA II). ${ }^{27,28}$

During the clinical trials, six deaths were observed in the OCR-exposed patients. One of these subjects was included in the Phase II trial and exposed to a high dose of OCR $(2,000 \mathrm{mg}) .{ }^{27}$ In this case, the death was due to an acute onset of thrombotic microangiopathy, occurred at week 14, and a correlation with the drug was not excluded. Another patient who was enrolled in the OPERA II trial died from suicide. ${ }^{28}$ The remaining four patients who died during the ORATORIO study died from pulmonary embolism, pneumonia, pancreatic carcinoma, and aspiration pneumonia. ${ }^{34}$

\section{Conclusion}

All clinical trials comparing OCR versus placebo or interferon beta have shown the superiority of this monoclonal antibody in many clinical and neuroimaging aspects. These studies are important for patients with RRMS, as these present a new therapeutic option. However, OCR is even more important for PPMS subjects, as it is the first drug to be approved for use in patients with this phenotype of MS. Given the strict inclusion criteria in the Phase III trial for patients with PPMS, the generalization of OCR treatment usefulness to patients without these features needs further clarification. Moreover, in both PP and RR patients, the impact of OCR in reducing the progression of brain atrophy was very small, $27,28,34$ and this result once again suggests that its prevalent action is on the inflammatory aspect of the disease, rather than on neurodegeneration itself. Indeed, it seems that the drug is not effective in slowing the neurodegenerative process.

As for all new drugs, only continuous observation can provide information about safety, in particular regarding rare adverse events such as malignancies and opportunistic infections, and regarding the exposure in a time of less than 6 months from pregnancy. A particular attention should be addressed on malignancies, as no clear data can be derived from clinical trials.

There are some interesting topics that need to be investigated.

First of all, there is limited experience with repetitive infusions of OCR, and more generally, with long-lasting B-cell depletion. This is true regarding both the efficacy and safety of OCR. In fact, the duration of clinical trials is limited, and a maximum number of infusions that can be administered have not been established.

Moreover, OCR will be used in clinical practice in both naïve patients and in those shifting from a second- or first-line treatment for MS. Unfortunately, no safety data are available about switching from and to OCR. To date, the possibility of rebound of disease activity after OCR discontinuation has not been described.

The continuous record of data derived from worldwide clinical practice will be very important in answering these issues. For this purpose, real-life registries for recording both the adverse events and the pregnancies that occurred with exposure to the drug should be created.

\section{Disclosure}

The authors report no conflicts of interest in this work.

\section{References}

1. Lublin FD, Reingold SC, Cohen JA, et al. Defining the clinical course of multiple sclerosis. Neurology. 2014;83(3):278-286.

2. Kurtzke JF. Rating neurologic impairment in multiple sclerosis: an expanded disability status scale (EDSS). Neurology. 1983;33(11): 1444-1452.

3. Brück W. The pathology of multiple sclerosis is the result of focal inflammatory demyelination with axonal damage. J Neurol. 2005;252(Supp1 5): $\mathrm{v} 3-\mathrm{v} 9$.

4. Esiri MM. Immunoglobulin-containing cells in multiple-sclerosis plaques. Lancet. 1977;2(8036):478.

5. Prineas JW, Connell F. The fine structure of chronically active multiple sclerosis plaques. Neurology. 1978;28(9 Pt 2):68-75.

6. Serafini B, Rosicarelli B, Magliozzi R, et al. Detection of ectopic B-cell follicles with germinal centers in the meninges of patients with secondary progressive multiple sclerosis. Brain Pathol. 2004;14(2):164-174.

7. Kabat EA, Glusman M, Knaub V. Immunochemical estimation of albumin and gamma globulin in normal and pathological cerebrospinal fluid. Am J Med. 1948;4:653-662. 
8. Yu X, Burgoon M, Green M, et al. Intrathecally synthesized IgG in multiple sclerosis cerebrospinal fluid recognizes identical epitopes over time. J Neuroimmunol. 2011;240-241:129-136.

9. Walsh MJ, Tourtellotte WW. Temporal invariance and clonal uniformity of brain and cerebrospinal $\operatorname{IgG}, \operatorname{IgA}$, and $\operatorname{IgM}$ in multiple sclerosis. J Exp Med. 1986;163(1):41-53.

10. Greenfield AL, Hauser SL. B cell therapy for multiple sclerosis: entering an era. Ann Neurol. Epub 2017 Dec 15.

11. Steri M, Orrù V, Idda ML, et al. Overexpression of the cytokine BAFF and autoimmunity risk. $N$ Engl J Med. 2017;376(17):1615-1626.

12. Mackay F, Schneider P, Rennert P, et al. BAFF AND APRIL: a tutorial on B cell survival. Annu Rev Immunol. 2003;21:231-264.

13. Mameli G, Cossu D, Caggiu E, et al. Soluble BAFF level is not correlated to Mycobacterium avium subspecies paratuberculosis antibodies and increases after interferon- $\beta$ therapy in multiple sclerosis patients. J Mol Neurosci. 2016;60(1):91-93.

14. Mameli G, Cocco E, Frau J, et al. Serum BAFF levels, methypredsinolone therapy, Epstein-Barr virus and Mycobacterium avium subsp. paratuberculosis infection in multiple sclerosis patients. Sci Rep. 2016;6:29268.

15. Dargahi N, Katsara M, Tselios T, et al. Multiple sclerosis: immunopathology and treatment update. Brain Sci. 2017;7(7). pii: E78.

16. Klein C, Lammens A, Schafer W, et al. Epitope interactions of monoclonal antibodies targeting CD20 and their relationship to functional properties. MAbs. 2013;5(1):22-33.

17. Gelfand JM, Cree BAC, Hauser SL. Ocrelizumab and other CD20 ${ }^{+}$ B-cell-depleting therapies in multiple sclerosis. Neurotherapeutics. 2017; 14(4):835-841.

18. Stashenko P, Nadler LM, Hardy R, et al. Characterization of a human B lymphocyte-specific antigen. J Immunol. 1980;125(4):1678-1685.

19. Anderson D, Grillo-Lopez A, Varns C, et al. Targeted anti-cancer therapy using rituximab, a chimaeric anti-CD20 antibody (IDEC-C2B8) in the treatment of non-Hodgkin's B-cell lymphoma. Biochem Soc Trans. 1997;25(2):705-708.

20. Clynes R, Towers T, Presta L, et al. Inhibitory Fc receptors modulate in vivo cytotoxicity against tumor targets. Nat Med. 2000;6(4): 443-446.

21. Mease P. B cell-targeted therapy in autoimmune disease: rationale, mechanisms, and clinical application. J Rheumatol. 2008;35:1245-1255.

22. Reff M, Carner K, Chambers K, et al. Depletion of B cells in vivo by a chimeric mouse human monoclonal antibody to CD20. Blood. 1994;83: 435-445.

23. Holley JE, Bremer E, Kendall AC, et al. CD20+ inflammatory T-cells are present in blood and brain of multiple sclerosis patients and can be selectively targeted for apoptotic elimination. Mult Scler Relat Disord. 2014;3(5):650-658.
24. Li R, Rezk A, Miyazaki Y, et al. Proinflammatory GM-CSFproducing B cells in multiple sclerosis and B cell depletion therapy. Sci Transl Med. 2015;7(310):310ra166.

25. Howell OW, Reeves CA, Nicholas R, et al. Meningeal inflammation is widespread and linked to cortical pathology in multiple sclerosis. Brain. 2011;134(Pt 9):2755-2771.

26. Magliozzi R, Howell OW, Reeves C, et al. A gradient of neuronal loss and meningeal inflammation in multiple sclerosis. Ann Neurol. 2010; 68(4):477-493.

27. Kappos J, Li D, Calabresi PA, et al. Ocrelizumab in relapsing-remitting multiple sclerosis: a phase 2, randomised, placebo-controlled, multicentre trial. Lancet. 2011;378(9805):1779-1787.

28. Hauser SL, Bar-Or A, Comi G, et al. Ocrelizumab versus interferon beta-1a in relapsing multiple sclerosis. $N$ Engl J Med. 2017;376(3): $221-234$.

29. Ocrevus (OCR) [product information]. South San Francisco, CA: Genetech, Inc; 2017.

30. Weber MS, Prod'homme T, Patarroyo JC, et al. B-cell activation influences $\mathrm{T}$ cell polarization and outcome of anti-CD20 B-cell depletion in central nervous system autoimmunity. Ann Neurol. 2010;68(3): 369-383.

31. Graves J, Vinayagasundaram U, Mowry EM, et al. Effects of rituximab on lymphocytes in multiple sclerosis and neuromyelitis optica. Multiple sclerosis and related disorders. 2014;3(2):244-252.

32. Hauser SL, Waubant E, Arnold DL, et al. B-cell depletion with rituximab in relapsing-remitting multiple sclerosis. $N$ Engl J Med. 2008; 358(7):676-688

33. Rahmanzadeh R, Weber MS, Bruck W, et al. B cells in multiple sclerosis therapy-A comprehensive review. Acta Neurol Scand. Epub 2018 Mar 7.

34. Montalban X, Hauser SL, Kappos L, et al. Ocrelizumab versus placebo in primary progressive multiple sclerosis. N Engl J Med. 2017;376(3): 209-220.

35. Hawker K, O’Connor P, Freedman MS, et al. Rituximab in patients with primary progressive multiple sclerosis: results of a randomized doubleblind placebo-controlled multicenter trial. Ann Neurol. 2009;66(4): 460-471.

36. Emery P, Rigby W, Tak PP, et al. Safety with OCR in rheumatoid arthritis: results from the OCR phase III program. PLoS One. 2014;9(2) e87379.

37. Fiore K. Genentech Reports PML Case with Ocrelizumab - Occurred in patient transitioning from natalizumab. Available from: https:// www.medpagetoday.com/neurology/multiplesclerosis/65537. Accessed May 24, 2017
Neuropsychiatric Disease and Treatment

\section{Publish your work in this journal}

Neuropsychiatric Disease and Treatment is an international, peerreviewed journal of clinical therapeutics and pharmacology focusing on concise rapid reporting of clinical or pre-clinical studies on a range of neuropsychiatric and neurological disorders. This journa is indexed on PubMed Central, the 'PsycINFO' database and CAS,

\section{Dovepress}

and is the official journal of The International Neuropsychiatric Association (INA). The manuscript management system is completely online and includes a very quick and fair peer-review system, which is all easy to use. Visit http://www.dovepress.com/testimonials.php to read real quotes from published authors. 